\title{
Resonant relaxation in protoplanetary disks
}

\author{
Scott Tremaine \\ Princeton University Observatory \\ Peyton Hall, Princeton, NJ 08544
}

\begin{abstract}
Resonant relaxation is a novel form of two-body relaxation that arises in nearly Keplerian disks such as protoplanetary disks. Resonant relaxation does not affect the semimajor axes of the particles, but enhances relaxation of particle eccentricities and inclinations. The equilibrium state after resonant relaxation is a Rayleigh distribution, with the mean-square eccentricity and inclination inversely proportional to mass. The rate of resonant relaxation depends strongly on the precession rate of the disk. If the precession due to the disk's self-gravity is small compared to the total precession, then the relaxation is concentrated near the secular resonance between each pair of interacting bodies; on the other hand if the precession rate is dominated by the disk's self-gravity then relaxation occurs through coupling to the large-scale low-frequency $m=1$ normal modes of the disk. Depending on the disk properties, resonant relaxation may be either stronger or weaker than the usual non-resonant relaxation.
\end{abstract}

\section{Introduction}

The formation of planets from a disk of planetesimals is largely determined by two closely related processes: physical collisions and gravitational relaxation (e.g. Stewart and Wetherill 1988, Lissauer and Stewart 1993). Collisions result from close two-body encounters and drive the evolution of the mass spectrum of the planetesimals, while relaxation arises mainly from distant two-body encounters and drives the evolution of their phase-space distribution. The relative rates of the two processes are determined by the Safronov number, $\theta \sim\left(v_{e} / v_{r}\right)^{2}$, where $v_{e}$ is the surface escape speed and $v_{r}$ is the rms random velocity of the planetesimals; relaxation dominates when $\theta \gg 1$. Gravitational relaxation has two main effects on the random velocity of a particle in a disk: scattering drives a stochastic random walk towards larger values of $v_{r}$, while dynamical friction damps $v_{r}$. In general, scattering dominates for small particles while dynamical friction dominates for massive ones, as expected by equipartition. 
The usual derivation of the rate of gravitational relaxation treats the evolution of the particle orbit as a sequence of uncorrelated two-body encounters with other particles. Such derivations neglect the effect of the gravitational field of the central star, but should be accurate in the early stages of planetesimal accumulation, so long as $v_{r} \gg n r_{H}$, where $n$ is the mean motion and $r_{H}=r\left(m / M_{\star}\right)^{1 / 3}$ is the Hill radius of the particle; here $m$ and $M_{\star}$ are the masses of the particle and the central star, and $r$ is the orbital radius (Ida 1990).

This papers investigates a qualitatively different type of gravitational relaxation, resonant relaxation, which we have already investigated in the context of spherical stellar systems (Rauch and Tremaine 1996). Resonant relaxation arises in addition to the usual ("nonresonant") relaxation discussed above, when the potential in which the particles orbit is nearly Keplerian. To introduce the concept, let us imagine a time-exposure of a particulate Keplerian disk, in which the exposure time is much longer than the orbital period $t_{\text {orb }}$ but shorter than the characteristic precession time $t_{\text {prec }}$. Each particle appears in the photograph as an eccentric ellipse or wire. These wires exert torques on one another which remain roughly constant for $\sim t_{\text {prec }}$; however, after several times $t_{\text {prec }}$ the configuration of the wires and the resulting torques will be quite different. These torques cause the angular momentum vectors of the particles - and thus their eccentricities and inclinations - to random walk, with the duration of one "step" in the walk being roughly $t_{\text {prec }}$. However, the energies or semimajor axes are unaffected because the potential from the wires is nearly stationary. A closely related process is resonant friction, which damps the eccentricities and inclinations of massive particles without affecting their semimajor axes. The combined effects of resonant relaxation and resonant friction drive the phase-space distribution of particles towards the maximum-entropy state consistent with fixed particle energies and total angular momentum. The main goal of the paper is to estimate the resonant rates of excitation and damping of protoplanet eccentricities, to compare these briefly with the non-resonant rates, and to demonstrate that in some cases resonant relaxation dominates the eccentricity evolution.

Resonant relaxation is a close cousin of secular perturbation theory in celestial mechanics, which likewise averages the Hamiltonian over times much longer than $t_{\text {orb }}$. Thus an alternative name for the process we are examining would be the oxymoron "secular relaxation".

Nearly Keplerian disks can be parametrized by two dimensionless numbers

$$
S(r) \equiv-\frac{g(r)}{n(r)} \frac{M_{\star}}{\pi r^{2} \Sigma(r)}=-\frac{g(r) n(r) r}{\pi G \Sigma(r)}, \quad Q(r) \equiv 1.07 \frac{\sigma_{r}}{n(r) r} \frac{M_{\star}}{\pi r^{2} \Sigma(r)}
$$

here $g=\dot{\varpi}$ is the apsidal precession rate, $\Sigma$ is the surface density of the disk, and $\sigma_{r}$ is the rms radial velocity, related to the mean-square eccentricity by $\sigma_{r}^{2}=\frac{1}{2} r^{2} n^{2}\left\langle e^{2}\right\rangle$. The second number is Toomre's $Q$-parameter (Binney and Tremaine 1987) and $Q>1$ is required for 
axisymmetric stability. If the precession rate is determined by the self-gravity of the disk, then generally $S \sim 1$. For example, a disk with a power-law surface density $\Sigma(r) \propto r^{-\beta}$ has

$$
S=2 \frac{\Gamma\left(2-\frac{1}{2} \beta\right) \Gamma\left(\frac{1}{2}+\frac{1}{2} \beta\right)}{\Gamma\left(\frac{3}{2}-\frac{1}{2} \beta\right) \Gamma\left(\frac{1}{2} \beta\right)}, \quad 0<\beta<3 ;
$$

in this case $S$ is independent of radius and varies only between 1 and 1.0942 in the range $1 \leq \beta \leq 2$.

More generally, if there are other sources of precession then $|S| \gg 1$. In the rings of Saturn and Uranus, where the precession is dominated by the planetary quadrupole moment, $S \sim-10^{5}$. In protoplanetary disks containing a thin disk of planetesimals embedded in a thicker disk of gas, $S$ will be roughly the ratio of gas mass to planetesimal mass, $S \sim 10^{2}$. We shall find that resonant relaxation is quite different in disks with $S \sim 1$ and $|S| \gg 1$.

To simplify the discussion, we shall focus on the effects of resonant relaxation on planets, that is, on bodies much larger than the planetesimals with which they are interacting. Also, we shall concentrate on resonant relaxation of the eccentricities, and generally ignore inclination relaxation except for a brief discussion in $\$ 6$.

The equilibrium distribution of the particle orbital elements in a resonantly relaxed disk is determined in 32 . Estimating the rate of resonant relaxation is a more challenging task. A variety of tools can be used for this purpose, depending on the properties of the disk. We shall employ secular perturbation theory in $\delta$ 跑 to provide analytic estimates of the rate of resonant relaxation and resonant friction in disks. We carry out similar calculations using WKB density-wave theory in $\S 凹$, and numerical normal-mode calculations in $\$ 5$. The results are summarized and discussed in $\$ 6$.

\section{Thermodynamic equilibrium}

The equilibrium eccentricity and inclination distribution of the particles in a resonantly relaxed disk can be determined by the methods of statistical mechanics. On timescales longer than the resonant relaxation time, the disk should be in the maximum-entropy state consistent with its total angular momentum and energy and mass distribution. Thus the equilibrium phase-space distribution function is (Rauch and Tremaine 1996)

$$
f(\mathbf{r}, \mathbf{v}, m)=w(E, m) \exp (m \mathbf{b} \cdot \mathbf{J}),
$$

where $E=\frac{1}{2} v^{2}-G M_{\star} / r, \mathbf{J}=\mathbf{r} \times \mathbf{v}$, and $\mathbf{b}$ and $w(E, m)$ are determined implicitly by the total angular momentum and the distribution of particles in mass and energy, both of which 
are conserved We orient the coordinate system so that $\mathbf{b}=b \hat{\mathbf{e}}_{z}$ and introduce the actions $\left(J_{c}, J, J_{z}\right)$, where $J_{c}=\left(G M_{\star} a\right)^{1 / 2}$ is the specific angular momentum of a circular orbit with semimajor axis $a, J=J_{c}\left(1-e^{2}\right)^{1 / 2}$ is the specific angular momentum, $J_{z}=J \cos I$ is the $z$-component of angular momentum, and $e$ and $I$ are the eccentricity and inclination. The conjugate angles are the mean anomaly, argument of perihelion, and longitude of ascending node (e.g. Brouwer and Clemence 1961). Then we have

$$
f(\mathbf{r}, \mathbf{v}, m)=w\left[E\left(J_{c}\right), m\right] \exp \left(m b J_{z}\right)
$$

where $E\left(J_{c}\right)=-\frac{1}{2}\left(G M_{\star}\right)^{2} / J_{c}^{2}$. The advantage of this form is that the phase-space volume element is simply $(2 \pi)^{3} d J_{c} d J d J_{z}$, so the density of particles in action space is given by

$$
d N=(2 \pi)^{3} w\left[E\left(J_{c}\right), m\right] \exp \left(m b J_{z}\right) d J_{c} d J d J_{z} .
$$

If the eccentricities and inclinations are small we may approximate $J$ and $J_{z}$ by $J_{c}\left(1-\frac{1}{2} e^{2}\right)$ and $J_{c}\left(1-\frac{1}{2} e^{2}-\frac{1}{2} I^{2}\right)$, so

$$
d N=W\left(J_{c}, m\right) \exp \left[-\frac{1}{2} m b L\left(e^{2}+I^{2}\right)\right] d J_{c} d e^{2} d I^{2},
$$

where $W\left(J_{c}, m\right)=2 \pi^{3} w\left[E\left(J_{c}\right), m\right] J_{c}^{2} \exp \left(m b J_{c}\right)$. This is the Rayleigh distribution (e.g. Lissauer and Stewart 1993), with the further constraint that the mean-square eccentricity and inclination at different semimajor axes and masses are related by $\left\langle e^{2}\right\rangle,\left\langle I^{2}\right\rangle \propto 1 /\left(m a^{1 / 2}\right)$.

\section{Secular perturbation theory}

We examine the relaxation of a planet of mass $m_{P}$, semimajor axis $a_{P}$, mean motion $n_{P}$, eccentricity $e_{P} \ll 1$ and longitude of periapsis $\varpi_{P}$. We shall also use the complex eccentricity $z_{P} \equiv e_{P} \exp \left(i \varpi_{P}\right)$. The disk is axisymmetric and composed of $N$ planetesimals with masses $m_{j}$ and orbital elements $a_{j}, n_{j}, e_{j}, \varpi_{j}, z_{j}, j=1, \ldots, N$. The planet is assumed to be much more massive than the planetesimals, $m_{j} \ll m_{P} \ll M_{\star}$. Kepler's law states that $n_{j}^{2} a_{j}^{3}=n_{P}^{2} a_{P}^{3}=G M_{\star}$.

We assume that the eccentricities are small in the sense that $e_{P}, e_{j} \ll\left|a_{j}-a_{P}\right| / a_{P}$. Then the secular evolution of the complex eccentricity is described by Lagrange's equations

\footnotetext{
${ }^{1}$ In contrast, the equilibrium distribution function for non-resonant relaxation is

$$
f(\mathbf{r}, \mathbf{v}, m)=w(m) \exp (-m \beta E+m \mathbf{b} \cdot \mathbf{J}),
$$

where $\beta$ and $\mathbf{b}$ are constants. This cannot be achieved in a Keplerian potential, since it requires that the density diverges as $\exp \left(G M_{\star} m \beta / r\right)$ as $r \rightarrow 0$.
} 
(Brouwer and Clemence 1961):

$$
\begin{aligned}
\dot{z}_{P} & =i g_{P} z_{P}-i \sum_{j=1}^{N} A_{P j} z_{j} \\
\dot{z}_{k} & =i g\left(a_{k}\right) z_{k}-i A_{k P} z_{P}-i \sum_{\substack{j=1 \\
j \neq k}}^{N} A_{k j} z_{j} .
\end{aligned}
$$

Here

$$
A_{k j}=\frac{2 G m_{j}}{n_{k} a_{k}^{2}} P_{k j}, \quad P_{k j}=P_{j k}=\frac{\alpha b_{3 / 2}^{(2)}(\alpha)}{8 \max \left(a_{k}, a_{j}\right)}, \quad \alpha=\frac{\min \left(a_{k}, a_{j}\right)}{\max \left(a_{k}, a_{j}\right)} .
$$

The function $g\left(a_{k}\right)=\dot{\varpi}_{k}$ and $g_{P}=\dot{\varpi}_{P}$ are the rates of apsidal precession of the disk and the planet; we defer a discussion of these until $\$ 3.3$. The Laplace coefficient $b_{s}^{(m)}(\alpha)$ is defined by

$$
b_{s}^{(m)}(\alpha)=\frac{2}{\pi} \int_{0}^{\pi} \frac{\cos m \phi d \phi}{\left(1-2 \alpha \cos \phi+\alpha^{2}\right)^{s}},
$$

and we will use the result that

$$
b_{3 / 2}^{(m)}(\alpha) \simeq \frac{2}{\pi(1-\alpha)^{2}} \quad \text { as } \alpha \rightarrow 1
$$

\subsection{Resonant relaxation}

Relaxation arises from the stochastic forces exerted on the planet by the planetesimal orbits. We concentrate first on disks in which the surface density is low, in the sense that $|S| \gg 1$ (cf. eq. 11). In this case we can drop the term $-i \sum_{j \neq k} A_{k j} z_{j}$ which represents mutual perturbations among planetesimals from the second of equations (8). We also drop the term $-i A_{k P} z_{P}$ which represents the forces from the planet on the planetesimals. Thus the planetesimal eccentricities satisfy $z_{k}(t)=z_{k}(0) \exp \left[i g\left(a_{k}\right) t\right]$, where $z_{k}(0)$ is the initial eccentricity. Substituting this result into the first of equations (8), and assuming that the initial planet eccentricity $z_{P}(0)=0$, we obtain

$$
z_{P}(t)=\sum_{j} A_{P j} z_{j}(0) \frac{\exp \left[i g\left(a_{j}\right) t\right]-\exp \left(i g_{P} t\right)}{g_{P}-g\left(a_{j}\right)} .
$$

We now compute the rate of change of the mean-square planetary eccentricity $\left\langle e_{P}^{2}\right\rangle$, where $\langle\cdot\rangle$ denotes an ensemble average. For axisymmetric disks the longitude of periapsis is uniformly distributed, so $\left\langle z_{j}^{*}(0) z_{k}(0)\right\rangle=0$ if $j \neq k$. Using this result we find

$$
\frac{d}{d t}\left\langle e_{P}^{2}\right\rangle=\left\langle z_{P}^{*} \frac{d z_{P}}{d t}\right\rangle+\left\langle z_{P} \frac{d z_{P}^{*}}{d t}\right\rangle=2 \sum_{j} A_{P j}^{2}\left\langle\left|z_{j}(0)\right|^{2}\right\rangle \frac{\sin \left[g_{P}-g\left(a_{j}\right)\right] t}{g_{P}-g\left(a_{j}\right)} .
$$


At large times we may use the relation $\lim _{t \rightarrow \infty} \sin (x t) / x=\pi \delta(x)$, where $\delta(x)$ is the Dirac delta-function; we also replace the sum over particles by an integral over semimajor axis. Thus

$$
\frac{d}{d t}\left\langle e_{P}^{2}\right\rangle=\frac{\pi^{2}}{4} n_{P}^{2}\left\langle e^{2}\right\rangle \frac{\left\langle m^{2}\right\rangle}{M_{\star}\langle m\rangle} \int \frac{\Sigma(a) a_{P} d a}{M_{\star}} \alpha^{3}\left[b_{3 / 2}^{(2)}(\alpha)\right]^{2} \delta\left[g(a)-g_{P}\right],
$$

where $\alpha=\min \left(a, a_{P}\right) / \max \left(a, a_{P}\right), \Sigma(a)$ is the surface density of the disk, and $\langle m\rangle$ and $\left\langle m^{2}\right\rangle$ are averages over the mass distribution. In this approximation, relaxation is entirely due to particles located at the secular resonance $a_{s}$ where $g\left(a_{s}\right)=g_{P}$.

\subsection{Resonant friction}

To evaluate the resonant friction on the planet, we return to Lagrange's equations (8) and once again drop the term $-i \sum_{j \neq k} A_{k j} z_{j}$ representing collective effects within the protoplanetary disk. Then if we assume that $z_{P}, z_{j} \propto \exp (i \omega t)$ we obtain the dispersion relation

$$
g_{P}-\omega-\sum_{j} \frac{A_{P j} A_{j P}}{g\left(a_{j}\right)-\omega}=0 .
$$

We replace the sum by an integral to obtain

$$
g_{P}-\omega-\frac{\pi}{8} n_{P} \frac{m_{P}}{M_{\star}} \int_{L} \frac{\Sigma(a) a d a}{M_{\star}} \alpha^{3}\left[b_{3 / 2}^{(2)}(\alpha)\right]^{2} \frac{n(a)}{g(a)-\omega}=0 .
$$

The subscript " $\mathrm{L}$ " is a reminder that the integral for $\operatorname{Im}(\omega)>0$ is the analytic continuation of the integral defined for $\operatorname{Im}(\omega)<0$ (the Landau contour). Equation (16) can be solved directly for the eigenfrequency of the coupled planet-planetesimal system, but it is easier and more informative to focus on the case where the coupling represented by the integral in equation (16) is weak enough that $\operatorname{Re}(\omega) \simeq g_{P}$. Then the rate of eccentricity growth or damping from resonant friction is given by

$$
\frac{d e_{P}^{2}}{d t}=-2 e_{P}^{2} \operatorname{Im}(\omega)=\frac{\pi}{4} e_{P}^{2} n_{P} \frac{m_{P}}{M_{\star}} \operatorname{Im} \int_{L} \frac{\Sigma(a) a d a}{M_{\star}} \alpha^{3}\left[b_{3 / 2}^{(2)}(\alpha)\right]^{2} \frac{n(a)}{g(a)-\omega} .
$$

Since the coupling is weak, $\operatorname{Im}(\omega)$ is small. For $\operatorname{Im}(\omega)$ small and negative, we may use the relation

$$
\lim _{\epsilon \rightarrow 0} \frac{\epsilon}{\epsilon^{2}+x^{2}}=\operatorname{sgn}(\epsilon) \pi \delta(x)
$$

to replace $\operatorname{Im}\left[(g-\omega)^{-1}\right]=\operatorname{Im} \omega /\left[\left(g-g_{P}\right)^{2}+(\operatorname{Im} \omega)^{2}\right]$ by $-\pi \delta\left(g-g_{P}\right)$. Thus we obtain

$$
\frac{d e_{P}^{2}}{d t}=-\frac{\pi^{2}}{4} e_{P}^{2} n_{P} \frac{m_{P}}{M_{\star}} \int \frac{\Sigma(a) a d a}{M_{\star}} n(a) \alpha^{3}\left[b_{3 / 2}^{(2)}(\alpha)\right]^{2} \delta\left[g(a)-g_{P}\right],
$$


and by Landau's prescription this is the correct expression for small values of $\operatorname{Im}(\omega)$ of either sign. Thus resonant friction always damps the planet's eccentricity ${ }^{2}$.

Equation (19) can be rewritten as

$$
\frac{d e_{P}^{2}}{d t}=-\frac{\pi^{2}}{4} e_{P}^{2} n_{P} \frac{m_{P}}{M_{\star}} \frac{\Sigma(a) a^{2}}{M_{\star}} \frac{n(a)}{|d g / d \log a|} \alpha^{3}\left[b_{3 / 2}^{(2)}(\alpha)\right]^{2},
$$

where all quantities are evaluated at the secular resonance $a_{s}$.

The planetary eccentricity is in equilibrium when $d\left\langle e_{P}^{2}\right\rangle / d t$ given by (14) plus $d e_{P}^{2} / d t$ given by (19) is zero. This occurs when

$$
e_{P}^{2}=\frac{a^{1 / 2}}{a_{P}^{1 / 2}} \frac{\left\langle m^{2}\right\rangle}{m_{P}\langle m\rangle}\left\langle e^{2}\right\rangle
$$

where the planetesimal parameters $\langle m\rangle,\left\langle m^{2}\right\rangle, a,\left\langle e^{2}\right\rangle$ are evaluated at the secular resonance. Equation (21) is closely related to the equipartition theorem (\$2).

There is an additional complication for a planetesimal disk embedded in a gaseous disk, as the gas disk may also have a secular resonance with the planet. The gas resonance will have a different location since the surface density of the gas disk is larger and its precession rate is modified by pressure. Equation (19) describes the resonant friction from the gas disk if $\Sigma(a)$ is replaced by the gas surface density and $g(a)$ by the precession rate of the gas elements.

\subsection{Precession rates}

The location of the secular resonance and hence the resonant relaxation rate is determined by the rate of apsidal precession of the planet and the disk particles. Precession can arise from several distinct sources. For example, the quadrupole moment of the central star induces precession at a rate

$$
g(a)=\frac{3 J_{2} R_{\star}^{2}\left(G M_{\star}\right)^{1 / 2}}{2 a^{7 / 2}},
$$

where $R_{\star}$ and $J_{2}$ are the radius and dynamical oblateness of the star. The quadrupole precession is negligible for most protoplanetary disks, although it dominates the precession in other systems such as planetary rings.

\footnotetext{
${ }^{2}$ This result holds even for spherical systems, so long as the velocity distribution is predominantly tangential (Rauch and Tremaine 1996).
} 
The planet induces precession in the planetesimals. In Lagrange's theory the resulting precession rate of an object with semimajor axis $a_{k}$ and mean motion $n_{k}$ is

$$
g\left(a_{k}\right)=\frac{2 G m_{P}}{n_{k} a_{k}^{2}} N_{k P}, \quad N_{k j}=N_{j k}=\frac{\alpha b_{3 / 2}^{(1)}(\alpha)}{8 \max \left(a_{k}, a_{j}\right)} .
$$

Strictly this is the free precession, i.e. the precession that would occur if the planet orbit were circular. There is also forced precession due to the planet's eccentricity, which corresponds to the term $-i A_{k P} z_{P}$ in the second of equations (8).

The planetesimals also induce precession in the planet and each other. By an obvious extension of equation (23), the free precession of an object at semimajor axis $a_{k}$ is given by

$$
g\left(a_{k}\right)=\sum_{j=1}^{N} \frac{2 G m_{j}}{n_{k} a_{k}^{2}} N_{k j}
$$

However, the correct interpretation of this result is subtle for a continuous disk. The subtleties are illustrated by the observation that equation (24) always predicts prograde free precession $(g(a)>0)$, whereas smooth, continuous, axisymmetric disks usually induce retrograde free precession (cf. eqs. 1 and 2). Moreover in the continuum limit $(N \rightarrow \infty$, $m_{j} \sim N^{-1}$ ), the precession rate predicted by equation (24) diverges as $N^{-1}$, whereas in a smooth continuous disk the precession rate is finite.

The discrepancy arises because equation (24) assumes that particle orbits do not cross (more precisely, that $e_{j}, e_{k} \ll\left|a_{j}-a_{k}\right| / a_{k}$ for all $j, k$ ). This assumption is correct for an object with small free eccentricity that orbits outside the disk (or even within a gap in the disk), but incorrect if the orbit is embedded in a continuous disk, no matter how small the free eccentricity. Equation (24) can be applied to a continuous disk only if the complex eccentricity $z(a)$ is a smooth function of semimajor axis, so orbits do not cross (i.e. if there are forced eccentricities but the free eccentricity is zero).

The simplest fix for this shortcoming is to "soften" the gravitational potential of the particles, by replacing the Laplace coefficient (10) by

$$
\widetilde{b}_{s}^{(m)}(\alpha)=\frac{2}{\pi} \int_{0}^{\pi} \frac{\cos m \phi d \phi}{\left(1-2 \alpha \cos \phi+\alpha^{2}+\epsilon^{2}\right)^{s}},
$$

where $\epsilon \ll 1$. In this case the expressions (9) and (23) for $P_{j k}$ and $N_{j k}$ are no longer valid, since they were derived using recursion relations among unsoftened Laplace coefficients (Brouwer and Clemence 1961); they must be replaced by

$$
P_{j k}=\frac{1}{4 \max \left(a_{k}, a_{j}\right)}\left[\frac{1}{2} \alpha^{2} \frac{d^{2} \widetilde{b}_{1 / 2}^{(1)}(\alpha)}{d \alpha^{2}}+\alpha \frac{d \widetilde{b}_{1 / 2}^{(1)}(\alpha)}{d \alpha}-\widetilde{b}_{1 / 2}^{(1)}(\alpha)\right]
$$




$$
N_{j k}=\frac{1}{4 \max \left(a_{k}, a_{j}\right)}\left[\frac{1}{2} \alpha^{2} \frac{d^{2} \widetilde{b}_{1 / 2}^{(0)}(\alpha)}{d \alpha^{2}}+\alpha \frac{d \widetilde{b}_{1 / 2}^{(0)}(\alpha)}{d \alpha}\right] .
$$

The precession rate predicted by equation (24) can then be applied to a continuous disk as long as the spacing between adjacent particles is small compared to the softening, $\mid a_{j}-$ $a_{j-1} \mid \ll \epsilon a_{j}$.

We shall also use $\epsilon$ more generally, to indicate the typical dimensionless thickness or epicycle size in the disk; for $|1-\alpha| \lesssim \epsilon$ the Lagrange equations (8) are not accurate.

Yet another possible source of precession is the gravity from the thick gas disk that generally envelops the planetesimal disk in the early stages of planet formation.

\subsection{The resonant relaxation rate}

To estimate the resonant relaxation rate we first determine the location of the secular resonance. We write the particle precession rate as $g(a)=g_{0}(a)+g_{1}(a)$, where $g_{0}$ is the smoothly varying precession rate due to the disk mass in gas and planetesimals, quadrupole moment of the central star, etc., while $g_{1}(a)$ is the precession due to the planet (eq. 23). The precession

rate of the planet itself is $g_{P}=g_{0}\left(a_{P}\right)$ (unless the disk properties change sharply at the planet's location, for example if there is a gap). We then expand $g_{0}(a)$ in a Taylor series around $a_{P}$; using equation (11) we then have

$$
a_{s}=a_{P}-\left(\frac{1}{2 \pi} \frac{m_{P}}{M_{\star}} \frac{n a^{2}}{d g_{0}(a) / d a}\right)_{a_{P}}^{1 / 3} .
$$

Of course, this result is only valid if $a_{P} \gg\left|a_{s}-a_{P}\right| \gg \epsilon a_{P}$; in other words the planet mass must be large enough that the secular resonance is separated by at least the typical epicycle size or disk thickness $\left(\sim \epsilon a_{P}\right)$. These constraints can be expressed approximately as

$$
S \epsilon^{3} \ll \frac{m_{P}}{m_{D}} \ll S
$$

where $m_{D}$ is the disk mass. The rates of resonant relaxation and friction are then given by equations (14) and (19):

$$
\begin{aligned}
\frac{1}{\left\langle e_{P}^{2}\right\rangle} \frac{d\left\langle e_{P}^{2}\right\rangle}{d t} & =\frac{(2 \pi)^{4 / 3}}{3} n \frac{\left\langle m^{2}\right\rangle}{M_{\star}\langle m\rangle} \frac{\Sigma a^{2}}{M_{\star}}\left|\frac{1}{n} \frac{d g_{0}}{d \log a}\right|^{1 / 3}\left(\frac{m_{P}}{M_{\star}}\right)^{-4 / 3}, \\
\frac{1}{e_{P}^{2}} \frac{d e_{P}^{2}}{d t} & =-\frac{(2 \pi)^{4 / 3}}{3} n \frac{\Sigma a^{2}}{M_{\star}}\left|\frac{1}{n} \frac{d g_{0}}{d \log a}\right|^{1 / 3}\left(\frac{m_{P}}{M_{\star}}\right)^{-1 / 3},
\end{aligned}
$$


where all quantities are evaluated at $a_{s}$. Note that smaller planets relax faster, because the resonant radius $a_{s}$ is closer.

These results have several limitations: (i) We have neglected the term $-i \sum_{j \neq k} A_{k j} z_{j}$ in equations (8), which describes collective effects arising from the self-gravity of the disk. (ii) The delta functions in equations (14) and (19) imply that as $t \rightarrow \infty$ all of the relaxation or drag comes from particles located exactly at the secular resonance. More generally, at large but finite times the relaxation or damping is due to the few particles concentrated within an ever-narrowing resonant band $\Delta a \sim\left|d a / d g_{d}\right| / t$; eventually there may be too few particles and too little mass in the resonant band for the formula to be valid. (iii) A related concern is that the eccentricities of the particles near resonance will become so large that nonlinear terms neglected in equations (\$) will limit the strength of the resonant interactions.

These concerns can be addressed using the complementary tool of density-wave theory, which treats the planetesimal disk as a continuous, collisionless, self-gravitating fluid.

\section{Density-wave theory}

The disk is taken to have surface density $\Sigma(r)$, radial velocity dispersion $\sigma_{r}(r)$, and epicycle frequency $\kappa(r)=n(r)-g(r)$, where as usual $n(r)$ and $g(r)$ are the mean motion and apsidal precession rate. We disturb the disk with a surface-density perturbation proportional to $\operatorname{Re}\left\{\exp \left[i\left(\int^{r} k(r) d r+m \phi-\omega t\right)\right]\right\}$, and seek a dispersion relation that relates the frequency $\omega$ to the radial and azimuthal wavenumbers $k$ and $m$. In the WKB or tight-winding limit $|k r| \gg 1$ the dispersion relation for a collisionless thin disk with a Rayleigh distribution of eccentricities is (e.g. Binney and Tremaine 1987)

$$
\kappa^{2}(r)-[m n(r)-\omega]^{2}-2 \pi G \Sigma(r)|k| F\left(\frac{\omega-m n(r)}{\kappa(r)}, \frac{k^{2} \sigma_{r}^{2}(r)}{\kappa^{2}(r)}\right)=0
$$

where

$$
F(s, x)=\frac{2}{x}\left(1-s^{2}\right) e^{-x} \sum_{j=1}^{\infty} \frac{I_{j}(x)}{1-s^{2} / j^{2}},
$$

and $I_{j}(x)$ is a modified Bessel function.

For brevity we shall focus on the predictions of density-wave theory for resonant friction; most of our results apply equally well to resonant relaxation. Thus we are interested in density waves excited by a planet on an eccentric orbit. The slowly varying non-axisymmetric component of the planet's gravitational potential has the form (e.g. Goldreich and Tremaine 
1980)

$$
\Phi(r, \phi, t)=\frac{G m_{P} e_{P}}{2 \max \left(a_{P}, r\right)}\left(1-\alpha \frac{d}{d \alpha}\right) b_{1 / 2}^{(1)}(\alpha) \cos \left[\phi-\varpi_{P}(t)\right],
$$

which corresponds to $m=1$ and frequency $\omega=g_{P}=\dot{\varpi}_{P}$. Since $|g|,\left|g_{P}\right| \ll n$ in this case, we may simplify equation (30) by neglecting terms of order $g^{2}$ :

$$
2 n(r)\left[g_{P}-g(r)\right]-2 \pi G \Sigma(r)|k| F\left(-1, \frac{k^{2} \sigma_{r}^{2}(r)}{n^{2}(r)}\right)=0,
$$

or

$$
g_{P}-g(r)-\frac{2 \pi G \Sigma(r)}{\sigma_{r}(r)}\left[\frac{e^{-x}}{x^{1 / 2}} I_{1}(x)\right]=0,
$$

where $x=k^{2} \sigma_{r}^{2} / n^{2}$. In the limit $x \ll 1$ this simplifies to

$$
g_{P}-g(r)-\frac{\pi G \Sigma(r)|k|}{n(r)}=0 \quad \text { or } \quad \frac{g_{P}}{g(r)}=1-\frac{|k r|}{S(r)}
$$

In the WKB approximation, the planet excites a density wave satisfying the dispersion relation (34). The wave is excited near the secular resonance, where $g_{P}=g(r)$ and $k \simeq 0$. The wave propagates in the region where $g(r)<g_{P}$; in the usual case where $g(r)<0$ and $|g(r)|$ decreases outwards, the wave propagates inwards as a leading spiral $(k<0)$; this is the long-wavelength branch of the dispersion relation. The wave becomes more and more tightly wound $(|k|$ increases) as it propagates. Eventually the wave encounters a turning point at $x=0.5841$ (the maximum of the quantity in square brackets in eq. 34) and thereafter propagates outward, continuing to become more tightly wound; this is the short-wavelength branch. The wave-propagation zone may be written

$$
0<g_{P}-g(r)<1.397 \frac{G \Sigma(r)}{\sigma_{r}(r)}=0.416 \frac{n(r)}{Q(r)},
$$

where $Q$ is defined in equation (1). As the wave returns to the secular resonance $|k| \rightarrow \infty$ and the wave is damped by wave-particle resonances.

The gravitational force from the leading spiral wave adds angular momentum - but almost no energy, since the wave pattern is nearly stationary in an inertial frame - to the planet orbit. Thus the planetary eccentricity is damped. The damping rate can be determined from formulae in Goldreich and Tremaine (1980), and turns out to be precisely the same as equation (19); this is a special case of the general result that the gravitational torques exerted on satellites by disks with a given surface-density distribution are largely independent of the nature of the collective effects in the disk (Goldreich and Tremaine 1979). 
This analysis addresses several of the concerns raised at the end of the previous section. In particular, in the WKB approximation the rate of resonant relaxation and friction is not affected by the self-gravity of the disk; thus equations (14) and (19) for the damping rate remain valid when collective effects are included. Moreover the collective effects ensure that a larger part of the disk is gravitationally coupled to the planet, not just the particles at the secular resonance: for example, equation (36) implies that most of the disk inside the secular resonance lies in the wave zone if $Q \lesssim n / g$; this is in contrast to waves in non-Keplerian disks or with $m \neq 1$, where the much stronger condition $Q \sim 1$ is needed for a large wave zone.

In fact, the principal limitation of the WKB analysis is that in many disks the collective effects are so strong that the WKB approximation is invalid. The "width" $\Delta a_{s}$ of the secular resonance is roughly given by $\int_{a_{s}-\Delta a_{s}}^{a_{s}}|k| d r=2 \pi$, where $k$ is given by the WKB dispersion relation (35). We find

$$
\Delta a_{s}=2 \pi\left(\frac{G \Sigma}{n|d g / d r|}\right)_{a_{s}}^{1 / 2}=2 \pi^{1 / 2} a_{s} S^{1 / 2}\left(\frac{d \log |g|}{d \log r}\right)_{a_{s}}^{-1 / 2} .
$$

The WKB analysis is only valid if the resonance width is much less than the distance between the planet and the secular resonance, which in turn requires

$$
\frac{m_{P}}{m_{D}} \gg S^{-1 / 2}
$$

The failure of the WKB approximation is even more complete in disks with $S \sim 1$. Since $\sigma_{r} \ll n r$ in thin disks, the dispersion relation (34) can only be satisfied if the quantity in square brackets is $\ll 1$. This in turn requires that $x \ll 1$ or $x \gg 1$; the latter condition corresponds to the short-wavelength branch of the dispersion relation, which is rapidly damped by wave-particle interactions (Landau damping). Thus we focus on the case $x \ll 1$, where the dispersion relation is given by (35), which is the same as for a cold disk (eq. 33 with $\sigma_{r} \rightarrow 0$ ). If $g_{P} / g$ and $S$ are of order unity, then (35) demands that $|k r| \sim 1$ so the WKB approximation is not valid, however small the surface density of the disk may be. This situation contrasts with the case of a non-Keplerian potential or an azimuthal wavenumber $m \neq 1$, where the WKB approximation $|k r| \gg 1$ becomes more and more accurate as the surface density becomes smaller. Since the WKB approximation is invalid, we can only make an order-of-magnitude analytic estimate of the resonant damping rate. To do this, we set $\Sigma\left(a_{s}\right) a_{s}^{2} \sim m_{D}$ in equation (20), where $m_{D}$ is the disk mass. Since the precession is dominated by the disk self-gravity, we set $|d g / d \log a| \sim n m_{D} / M_{\star}$. Setting the Laplace coefficient and $\alpha_{s}$ to unity and neglecting other factors of order unity we obtain

$$
\frac{1}{e_{P}^{2}} \frac{d e_{P}^{2}}{d t} \sim-n \frac{m_{P}}{M_{\star}} .
$$


The eccentricity damping rate is independent of the disk mass, except that when the disk mass becomes smaller than the planet mass the precession is dominated by the planet so that equation (39) is no longer valid.

For a more accurate determination of the rate of eccentricity damping in disks with $S \sim 1$, we must turn to numerical calculations.

\section{Excitation of normal modes}

The preceding discussion suggests that the large-scale, low-frequency, $m=1$ normal modes of thin disks with $S \sim 1$ are largely independent of the velocity dispersion (eq. 35). Thus we can determine the response of the disk to a planet using Lagrange's theory (eq. 8) as a discrete approximation to a continuous disk with complex eccentricity $z(a)$.

We first determine the normal modes. If the terms involving the planet are dropped from the second of equations (8), we have

$$
\dot{z}_{k}=i g\left(a_{k}\right) z_{k}-i \sum_{\substack{j=1 \\ j \neq k}}^{N} A_{k j} z_{j}
$$

where $g\left(a_{k}\right)$ is given by equation (24). This has solution

$$
z_{k}=\sum_{n=1}^{N} \frac{b_{n} c_{k}^{n}}{\psi_{k}} \exp \left(i \omega_{n} t\right)
$$

where $\psi_{k}=\left(m_{k} n_{k}\right)^{1 / 2} a_{k}, b_{n}$ is arbitrary, and $\omega_{n}$ and $\mathbf{c}^{n}=\left(c_{1}^{n}, \ldots, c_{N}^{n}\right)$ are the $n^{\text {th }}$ eigenvalue and eigenvector of the matrix $\mathbf{M}$ defined by

$$
M_{k j}=g\left(a_{j}\right) \delta_{k j}-\frac{\psi_{k}}{\psi_{j}}\left(1-\delta_{k j}\right) A_{k j} ;
$$

that is, $\left(\mathbf{M}-\omega_{n} \mathbf{I}\right) \mathbf{c}^{n}=0$. The matrix $\mathbf{M}$ is symmetric and real, so all of the eigenvalues $\omega_{n}$ are real, and the eigenvectors $\mathbf{c}^{n}$ can be chosen to be real and orthonormal, that is, $\mathbf{c}^{n} \cdot \mathbf{c}^{m}=\delta_{m n}$.

Now suppose that a planet is present, with eccentricity $z_{P} \propto \exp (i \omega t)$. Since the eigenvectors are complete, the forced response of the disk particles may be written in the form

$$
z_{k}=\sum_{n=1}^{N} \frac{b_{n} c_{k}^{n}}{\psi_{k}} z_{P}
$$


solving the second of equations (8) we find

$$
b_{n}=\frac{1}{\omega_{n}-\omega} \sum_{k=1}^{N} \psi_{k} c_{k}^{n} A_{k P}
$$

Using this result to eliminate $z_{j}$ from the first of equations (\$) we obtain the dispersion relation for $\omega$ :

$$
g_{P}-\omega-\sum_{n=1}^{N} \frac{1}{\omega_{n}-\omega} \sum_{j, k=1}^{N} \frac{\psi_{k}}{\psi_{j}} A_{P j} A_{k P} c_{j}^{n} c_{k}^{n}=0 .
$$

This can be contrasted to equation (15), which was derived by neglecting the self-gravity of the disk; in this case $c_{k}^{n}=\delta_{k n}, \omega_{n}=g\left(a_{n}\right)$, and equation (45) reduces to (15).

As in the discussion following equation (15) we concentrate on the case where the planet mass $m_{P}$ is small compared to the disk mass $m_{D}$. We may then proceed as in equations (15)-(19) to obtain

$$
\frac{1}{e_{P}^{2}} \frac{d e_{P}^{2}}{d t}=-2 \pi \sum_{n=1}^{N} \delta\left(\omega_{n}-g_{P}\right) \sum_{j, k=1}^{N} \frac{\psi_{k}}{\psi_{j}} A_{P j} A_{k P} c_{j}^{n} c_{k}^{n} .
$$

The eccentricity damping arises from resonances between the planet's precession frequency and the disk eigenfrequencies. In this derivation the spectral lines of the disk and planet have zero width, but in practice a number of mechanisms are likely to broaden these lines: (i) evolution of the planet's mass or semimajor axis during the planet-formation process; (ii) evolution of the disk mass or surface density due to infall or angular-momentum transport; (iii) damping of the disk normal modes from wave-particle resonances or other mechanisms; (iv) the planet's spectral line is broadened by $\mathrm{O}(\operatorname{Im} \omega)$ which is $\sim g_{P}\left(m_{P} / m_{D}\right)$ for disks with $S \sim 1$. Another route to a similar conclusion is to recall that the typical precession time $g^{-1}$ is likely to be $10^{3}-10^{4}$ yr (for a disk mass of $10^{-2}-10^{-3} M_{\odot}$ at a radius of $5 \mathrm{AU}$ ), and the likely formation time for the giant planets is $10^{5}-10^{6} \mathrm{yr}$, during which the disk parameters and planetary orbit evolve substantially. Thus we expect the precessional spectra of the disk and planet to be broadened by at least of order $1 \%$.

To provide a crude representation of this effect - and to help ensure that the damping rates we obtain are well-behaved - we shall broaden the delta function into a Lorentz function by replacing $\delta\left(g_{P}-\omega_{n}\right)$ by $\pi^{-1} h g_{P} /\left[\left(g_{P}-\omega_{n}\right)^{2}+h^{2} g_{P}^{2}\right]$, a replacement which is exact in the limit $h \rightarrow+0$; usually we pick $h=0.1$. The use of a Lorentzian rather than some other broadening function is arbitrary but plausible.

Figure 1 shows examples of damping rates computed by equation (46). We have examined two disk models: the first has sharp edges, with surface density

$$
\Sigma_{1}(r)= \begin{cases}\Sigma_{0}\left(r_{0} / r\right)^{1.5}, & r_{i}<r<r_{0} \\ 0 & \text { otherwise }\end{cases}
$$




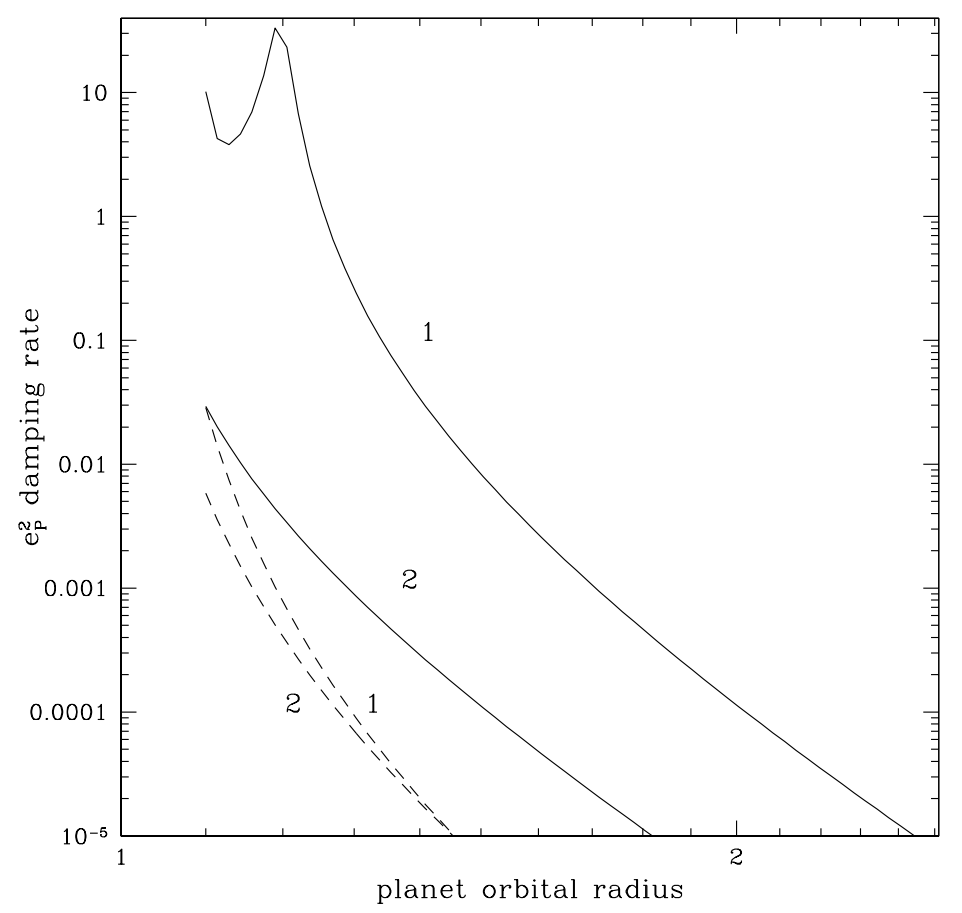

Fig. 1. - The damping rate $d \log \left(e_{P}^{2}\right) / d t$ for the eccentricity of a planet orbiting outside a Keplerian disk, as a function of the planet's semimajor axis. The curves labeled "1" are for a sharp-edged disk with surface density given by equation (47) and the curves labeled "2" are for a smooth disk (eq. 48). The solid curves include the effects of the disk self-gravity (eq. 46) and the dashed curves do not (eq. 19). The units are $G=M_{\star}=r_{0}=1$; the damping rate is proportional to the planet mass $m_{P}$ and is plotted for $m_{P}=1$. 
the second is smooth and is obtained by multiplying $\Sigma_{1}(r)$ by a window function in $\log r$ :

$$
\Sigma_{2}(r)=\Sigma_{1}(r) \sin ^{2}\left[\pi \log \left(r / r_{i}\right) / \log \left(r_{0} / r_{i}\right)\right] .
$$

We choose $r_{i}=0.1 r_{0}, h=0.1$, and the results are expressed in units in which $G=M_{\star}=$ $r_{0}=1$. The eccentricity damping rate is proportional to the planet mass $m_{P}$ and is plotted for $m_{P}=1$; in this approximation the damping rate is independent of the disk surface density $\Sigma_{0}$. The planet is assumed to orbit outside $r_{0}$, and the damping rate is plotted as a function of the planet's orbital radius. The solid curves are obtained from equation (46), which includes the effects of the disk's self-gravity, while the dashed curves do not (eq. 19).

The damping rate for the sharp-edged disk exhibits a resonant peak near an orbital radius of 1.2. The peak arises from a resonance between the planet's precession rate and the fundamental $m=1$ mode of the disk (i.e. the normal mode with zero nodes). At larger orbital radii the damping rate is determined by the tail of the Lorentz function associated with this and other normal modes (and thus depends on our arbitrary choice of a Lorentzian profile with width $h=0.1$ ).

The damping rate is lower for the smooth disk; in this case the eigenfrequencies of the disk are higher than the precession frequency of the planet, so the damping rate for a planet outside the disk is everywhere determined by the tails associated with each resonance.

These results can be re-stated by writing the heuristic formula (39) as

$$
\frac{1}{e_{P}^{2}} \frac{d e_{P}^{2}}{d t} \sim-f n \frac{m_{P}}{M_{\star}} ;
$$

then the dimensionless factor $f$ is approximately the same as the ordinate of Figure 1 for planets orbiting at semimajor axes near the disk edge. The important feature of Figure 1 is not the numerical damping rates, which depend on our arbitrary broadening function, but that even modest broadening leads to substantial damping over a large radius range.

Computing the eccentricity damping rate for a planet embedded in a disk is more complicated, since (i) the planetary precession rate and hence the damping will depend sensitively on whether there is an annular gap in the disk surrounding the planet; (ii) the planetary potential will have higher spatial frequencies and thus will interact with a richer set of disk normal modes; (iii) the approximation of small eccentricity is only valid if $e, e_{P} \ll$ $\left|a-a_{P}\right| / a_{P}$, and this is much more difficult to satisfy for nearby particles; (iv) non-resonant friction due to close encounters between the planet and disk particles will also play an important role in eccentricity evolution (see $\$$ 6 below). 


\section{Discussion}

We have investigated resonant gravitational relaxation, which arises in particle disks orbiting in a nearly Keplerian potential. Resonant relaxation does not affect semimajor axes, and tends to produce a Rayleigh distribution in eccentricity and inclination, with mean-square eccentricity or inclination inversely proportional to mass (eq. 7).

The nature of resonant relaxation depends strongly on whether apsidal precession is dominated by the self-gravity of the disk, a condition which is parametrized by $S(r)$ (eq. 1). If other mechanisms dominate the precession $(|S| \gg 1)$ then resonant relaxation requires that a secular resonance is present in the disk, which in turn requires $m_{P} / m_{D} \lesssim S$, where $m_{P}$ and $m_{D}$ are the planet and disk masses (eq. 28). If the resonance is present, the rate of damping of the planet's eccentricity from resonant friction is roughly (eq. 29)

$$
\frac{1}{e_{P}} \frac{d e_{P}}{d t} \sim-n\left(\frac{m_{D}}{M_{\star}}\right)^{4 / 3} S^{1 / 3}\left(\frac{m_{P}}{M_{\star}}\right)^{-1 / 3},
$$

and the rate of stochastic excitation of the eccentricity is

$$
\frac{1}{\left\langle e_{P}^{2}\right\rangle} \frac{d\left\langle e_{P}^{2}\right\rangle}{d t}=\frac{\left\langle m^{2}\right\rangle}{M_{\star}\langle m\rangle}\left(\frac{M_{\star}}{m_{P}}\right)\left|\frac{1}{e_{P}} \frac{d e_{P}}{d t}\right| .
$$

These formulae also require that (i) the planet mass is large enough that the secular resonance is separated from the planet by more than the characteristic disk thickness or epicycle size (eq. 28); (ii) the separation exceeds the characteristic width of the resonance caused by the disk self-gravity, $m_{P} / m_{D} \gtrsim S^{-1 / 2}$ (eq. 38).

If on the other hand the apsidal precession is determined by the disk mass $(S \sim 1)$, the planet interacts with the large-scale low-frequency normal modes of the disk rather than with particles near the secular resonance. Calculating the rate of resonant relaxation in this case is harder (cf. §5), especially if the planet is embedded in the disk. For heuristic purposes the rate of eccentricity decay from resonant friction may be written as (eq. 39)

$$
\frac{1}{e_{P}^{2}} \frac{d e_{P}^{2}}{d t} \sim-n \frac{m_{P}}{M_{\star}} .
$$

A key point is that the damping rate is independent of the disk mass $m_{D}$ so long as the disk mass determines the precession rate.

A major uncertainty in equation (52) is whether the planetary precession rate is in resonance with one of the disk eigenfrequencies; this usually requires either a continuous spectrum of eigenfrequencies or that dissipative or evolutionary processes broaden the discrete disk eigenfrequencies so that they overlap. Because the characteristic frequency of the 
disk modes is of order $n\left(m_{D} / M_{\star}\right) \ll n$, even slow evolutionary or damping processes cause considerable broadening. An important next step will be to understand the low-frequency, large-scale $m=1$ eigenmodes of nearly Keplerian disks.

For comparison, the non-resonant eccentricity damping rate for a planet embedded in a disk may be written as (Stewart and Wetherill 1988, Lissauer and Stewart 1993)

$$
\frac{1}{e_{P}^{2}} \frac{d e_{P}^{2}}{d t} \sim-n \frac{m_{P}}{M_{\star}} \frac{m_{D}}{M_{\star}}\left(\frac{v_{c}}{v_{r}}\right)^{4} \ln \Lambda,
$$

where $v_{r}$ is the rms random velocity of the planetesimals, $v_{c}$ is the circular speed, and $\ln \Lambda$ is the Coulomb logarithm. As described in $\S\left[1\right.$, this result is valid so long as $v_{r} \gg n r_{H}$ where $r_{H}$ is the planet's Hill radius (Ida 1990).

If a planet orbits at a distance $\Delta a$ from a narrow ring, the non-resonant damping rate becomes (Goldreich and Tremaine 1980, eq. 31)

$$
\frac{1}{e_{P}^{2}} \frac{d e_{P}^{2}}{d t} \sim-n \frac{m_{P}}{M_{\star}} \frac{m_{D}}{M_{\star}}\left(\frac{a_{p}}{|\Delta a|}\right)^{5} .
$$

These crude formulae suggest that either resonant or non-resonant eccentricity damping can dominate in a given disk, depending on the disk mass, velocity dispersion, and other parameters. In typical protoplanetary disks, non-resonant damping probably dominates if the planet is embedded in the disk. However if there is a gap $\Delta a / a_{P} \gtrsim\left(m_{D} / M_{\star}\right)^{1 / 5}$ then resonant damping may be stronger.

Resonant inclination relaxation is similar to resonant eccentricity relaxation. The principal difference is that the precession due to the self-gravity of the disk is $g \sim n m_{D} / M_{\star}\left(v_{c} / v_{r}\right)$, stronger by a factor of order $v_{c} / v_{r}$. Thus the minimum value of the parameter $|S| \sim v_{c} / v_{r} \gg$ 1.

Resonant relaxation is a complex process whose efficiency depends sensitively on properties such as the precession rate, the presence of gaps around planets, etc. (although it is much less sensitive to the rms random velocity in the disk than non-resonant relaxation). Moreover many of the tools that are usually employed to investigate disk relaxation (two-body scattering, Fokker-Planck approximation, Hill's problem, etc.) are too crude to describe resonant relaxation. Nevertheless this process is likely to play an important role in the evolution of a variety of nearly Keplerian disks, including both protoplanetary disks and other objects such as stellar disks surrounding black holes at the centers of galaxies.

I thank Kevin Rauch for comments and suggestions. This research was supported in part by NASA Grant NAG5-7310. 


\section{REFERENCES}

Binney, J. J., and Tremaine, S. 1987, Galactic Dynamics (Princeton: Princeton University Press)

Brouwer, D., and Clemence, G. M. 1961, Methods of Celestial Mechanics (New York: Academic Press)

Goldreich, P., and Tremaine, S. 1979, ApJ 233, 857

Goldreich, P., and Tremaine, S. 1980, ApJ 241, 425

Ida, S. 1990, Icarus 88, 129

Lissauer, J. J., and Stewart, G. R. 1993, in Protostars and Planets III, eds. Levy, E. H., Lunine, J. I., and Matthews, M. S. (Tucson: University of Arizona Press), 1061

Rauch, K. P., and Tremaine, S. 1996, NewA 1, 149

Stewart, G. R., and Wetherill, G. W. 1988, Icarus 74, 542 